\title{
Effects of Lorentz Symmetry Breaking and External Potential on a Relativistic Scalar Particle
}

\author{
Faizuddin Ahmed ${ }^{1}$ \\ National Academy Gauripur, Assam, 783331, India
}

\begin{abstract}
In this paper, a relativistic scalar particle under Lorentz symmetry breaking effects in the presence of a scalar potential is investigated. We introduce the scalar potential by modifying the mass via transformation $M \rightarrow M+S(r)$ in the wave equation and analyze the behaviour of a scalar particle. We see that the analytical solution to the KleinGordon equation can be achieved, and the energy eigenvalues and the wave function depends on the Lorentz symmetry breaking parameters as well as potential.
\end{abstract}

Keywords: Lorentz symmetry violation, Relativistic wave-equations, electric \& magnetic field, special function.

PACS Number(s): 03.65.Pm, 11.30.Cp, 11.30.Qc

\section{Introduction}

In the context of quantum systems, Lorentz symmetry violation (LSV) has been investigated by several authors, for example, in the nonrelativistic limit, the spectrum of hydrogen atom [1], the quantum Hall conductivity [2], the Aharonov-Bohm-Casher effect [3], neutral Dirac particle [4, 5, 6, 7], Rashbalike coupling [8, 6], Landau-type system subject to potential [9], geometric quantum phases $[4,5,10,11]$, and harmonic oscillator [12]; In the relativistic limit, relativistic scalar particle $[13,14,15,16]$, quantum oscillator

\footnotetext{
${ }^{1}$ faizuddinahmed15@gmail.com ; faiz4U.enter@rediffmail.com
} 
[17], the Landau-He-McKellar-Wilkens quantization and Dirac particle [18], Klein-Gordon oscillator [19], Dirac oscillator [20], and the geometric quantum phases [21, 22]. We investigate the relativistic quantum motion of scalar particles in under the effects of Lorentz symmetry breaking defined by a tensor $\left(K_{F}\right)_{\mu \nu \alpha \beta}$ out of the Standard Model Extension (SME) in the presence of a scalar potential. We analyze the effects of a radial electric field and a constant magnetic induced by the Lorentz symmetry violation. We see that the solution of the bound state to the Klein-Gordon equation can be obtained.

In the gauge sector of SME $[23,24,25,26]$, there are two types of Lorentzviolating contributions in the electromagnetic sector of SME that modifies the transport properties of space-time. These two types are called the CPTodd sector [23, 24] and the CPT-even sector [27, 28, 29, 30, 31, 32, 33]. The background of the Lorentz symmetry violation establishes by a tensor field can be introduced into the Klein-Gordon equation through a nonminimal coupling $\frac{g}{4}\left(K_{F}\right)_{\mu \nu \alpha \beta} F^{\mu \nu}(x) F^{\alpha \beta}(x)$, where $g$ is the coupling constant, $F_{\mu \nu}(x)$ is the electromagnetic field tensor, and $\left(K_{F}\right)_{\mu \nu \alpha \beta}$ is a dimensionless tensor. The tensor $\left(K_{F}\right)_{\mu \nu \alpha \beta}$ has the symmetries of the Riemann tensor $R_{\mu \nu \alpha \beta}$ and zero on double trace $\left(K_{F}\right)_{\mu \nu}^{\mu \nu}=0$, so it contains 19 independent real components (for details of this tensor, please see Refs. [23, 24, 29, 30, 31, 32, 33]). The current limits on the coefficients of the Lorentz symmetry violation can be found in details in Ref. [34].

The relativistic quantum dynamics of a scalar particle under the effects of LSV Refs. [23, 24, 8, 14, 15, 16, 17, 18, 19, 20] is given by

$$
p^{\mu} p_{\mu} \Psi+\frac{g}{4}\left(K_{F}\right)_{\mu \nu \alpha \beta} F^{\mu \nu}(x) F^{\alpha \beta}(x) \Psi=M^{2} \Psi,
$$

where $\Psi$ is the scalar wave function, and $M$ is the rest mass of the particle.

The structure of this paper is as follows: in section 2, we introduce the Lorentz symmetry violation defined by a tensor $\left(K_{F}\right)_{\mu \nu \alpha \beta}$ out of the Standard Model Extension. Then, we analyze the behaviour of a relativistic scalar particle in the presence of a scalar potential by solving the Klein-Gordon equation; in section 3, we present our conclusions. 


\section{Lorentz Symmetry Breaking Effects on a Relativistic Scalar Particle with potential}

We consider the Minkowski flat space-time in the cylindrical coordinates $(t, r, \phi, z)$

$$
d s^{2}=-d t^{2}+d r^{2}+r^{2} d \phi^{2}+d z^{2}
$$

where the ranges of the coordinates are $-\infty<(t, z)<\infty, r \geq 0$ and $0 \leq \phi \leq 2 \pi$.

For the geometry (2), the Klein-Gordon equation under the effects of the Lorentz symmetry violation becomes

$$
\begin{aligned}
& {\left[-\frac{\partial^{2}}{\partial t^{2}}+\frac{\partial^{2}}{\partial r^{2}}+\frac{1}{r} \frac{\partial}{\partial r}+\frac{\partial^{2}}{\partial z^{2}}+\frac{1}{r^{2}} \frac{\partial^{2}}{\partial \phi^{2}}\right] \Psi+\frac{g}{4}\left(K_{F}\right)_{\mu \nu \alpha \beta} F^{\mu \nu}(x) F^{\alpha \beta}(x) \Psi} \\
& =M^{2} \Psi
\end{aligned}
$$

Now using the properties of the tensor $\left(K_{F}\right)_{\mu \nu \alpha \beta}$ given in Refs. [29, 30, 31], we can rewrite $(3)$ in the following form :

$$
\begin{aligned}
& {\left[-\frac{\partial^{2}}{\partial t^{2}}+\frac{\partial^{2}}{\partial r^{2}}+\frac{1}{r} \frac{\partial}{\partial r}+\frac{1}{r^{2}} \frac{\partial^{2}}{\partial \phi^{2}}+\frac{\partial^{2}}{\partial z^{2}}\right] \Psi} \\
& +\left[-\frac{g}{2}\left(\kappa_{D E}\right)_{i j} E^{i} E^{j}+\frac{g}{2}\left(\kappa_{H B}\right)_{j k} B^{i} B^{j}-g\left(\kappa_{D B}\right)_{j k} E^{i} B^{j}\right] \Psi \\
& =M^{2} \Psi
\end{aligned}
$$

The known procedure in introducing a scalar potential into the Klein-Gordon equation was given in Ref. [35, 36], where the mass term is modified via transformation $M \rightarrow M+S(r)$, where $S(r)$ is a scalar potential. The scalar potentials of various kind have been used to obtain the solution of the bound state of the relativistic wave equations under LSV effects in Refs. $[14,16,17,18,19]$. In this work, we consider a scalar potential proportional to the inverse of the axial distance $\left(\propto \frac{1}{r}\right)[14,17,36]$, then, the mass term of the Klein-Gordon equation becomes

$$
M \rightarrow M+\frac{\eta_{c}}{r}
$$


where $\eta_{c}$ is a constant.

Let us consider a possible scenario of the Lorentz symmetry violation determined by the non-null components $\left(\kappa_{D E}\right)_{11}=$ const, $\left(\kappa_{D B}\right)_{13}=$ const with the field configuration given by $[5,16,19,20]$ :

$$
\vec{B}=B_{0} \hat{z} \quad, \quad \vec{E}=\frac{\lambda}{r} \hat{r}
$$

where $B_{0}>0, \hat{z}$ is a unit vector in the $z$-direction, $\lambda$ is the linear charge density, and $\hat{r}$ is the unit vector in the radial direction.

Hence, equation (4) using (5) and (6) becomes

$$
\begin{aligned}
& {\left[-\frac{\partial^{2}}{\partial t^{2}}+\frac{\partial^{2}}{\partial r^{2}}+\frac{1}{r} \frac{\partial}{\partial r}+\frac{1}{r^{2}} \frac{\partial^{2}}{\partial \phi^{2}}+\frac{\partial^{2}}{\partial z^{2}}\right] \Psi} \\
& +\left[-\frac{g}{2}\left(\kappa_{D E}\right)_{11} \frac{\lambda^{2}}{r^{2}}-\frac{g \lambda B_{0}}{r}\left(\kappa_{D B}\right)_{13}\right] \Psi=\left(M+\frac{\eta_{c}}{r}\right)^{2} \Psi .
\end{aligned}
$$

Since the metric $(2)$ is independent of $(t, \phi, z)$, let a possible total wave function is given by

$$
\Psi(t, r, \phi, z)=e^{i(-\varepsilon t+l \phi+k z)} \psi(r)
$$

where $E$ is the energy of the particle, $l=0, \pm 1, \pm 2, \ldots$ are the eigenvalues of the $z$-component of the angular momentum operator, and $k$ is the constant.

Substituting the solution (8) into the Eq. (7), we obtain the following radial wave-equation for $\psi(r)$ :

$$
\psi^{\prime \prime}(r)+\frac{1}{r} \psi^{\prime}(r)+\left[-\Lambda^{2}-\frac{j^{2}}{r^{2}}-\frac{\delta}{r}\right] \psi(r)=0,
$$

where

$$
\begin{aligned}
& \Lambda^{2}=M^{2}+k^{2}-\varepsilon^{2}, \\
& j=\sqrt{l^{2}+\eta_{c}^{2}+\frac{1}{2} g \lambda^{2}\left(\kappa_{D E}\right)_{11}} \\
& \delta=2 M \eta_{c}+g \lambda B_{0}\left(\kappa_{D B}\right)_{13} .
\end{aligned}
$$


Let us now perform a change of variable by $x=2 \Lambda r$. Then, from Eq. (9) we have

$$
\psi^{\prime \prime}(x)+\frac{1}{x} \psi^{\prime}(x)+\left(-\frac{1}{4}-\frac{j^{2}}{x^{2}}-\frac{\delta}{2 \Lambda x}\right) \psi(x)=0,
$$

By analysing the asymptotic behaviour of the Eq. (11) at $x \rightarrow 0$ and at $x \rightarrow \infty$, we have a solution to the Eq. (11) that can be written in terms of an unknown function $F(x)$ as

$$
\psi(x)=x^{j} e^{-\frac{x}{2}} F(x)
$$

Thereby, substituting the solution (12) into the Eq. (11), we obtain the following equation:

$$
x F^{\prime \prime}(x)+(1+2 j-x) F^{\prime}(x)+\left(-\frac{\delta}{2 \Lambda}-j-\frac{1}{2}\right) F(x)=0,
$$

which is called as the confluent hypergeometric equation [37, 38]; thus, the function $F(x)$ is the confluent hyper-geometric function, that is, $F(x)=$ ${ }_{1} F_{1}\left(j+\frac{1}{2}+\frac{\delta}{2 \Lambda}, 2 j+1, x\right)$. It is well-known that the confluent hyper-geometric series becomes a polynomial of degree $n$ when $j+\frac{1}{2}+\frac{\delta}{2 \Lambda}=-n[37,38]$ where, $n=0,1,2, .$.

Thus, we have obtained the following energy eigenvalues $\varepsilon_{n, l}$ expression:

$$
\varepsilon_{n, l}= \pm \sqrt{M^{2}+k^{2}-\frac{\left(M \eta_{c}+\frac{g \lambda}{2}\left(\kappa_{D B}\right)_{13}\right)^{2}}{\left(n+\frac{1}{2}+\sqrt{\left.l^{2}+\eta_{c}^{2}+\frac{1}{2} g \lambda^{2}\left(\kappa_{D E}\right)_{11}\right)^{2}}\right.}}
$$

The radial wave function is given by

$$
\psi_{n, l}(x)=x^{\sqrt{l^{2}+\eta_{c}^{2}+\frac{1}{2} g \lambda^{2}\left(\kappa_{D E}\right)_{11}}} e^{-\frac{x}{2}}{ }_{1} F_{1}\left(j+\frac{1}{2}+\frac{\delta}{2 \Lambda}, 2 j+1, x\right) .
$$

Hence, Eq. (14) gives the allowed values of energy level of a relativistic scalar particle under the effects under the effects of the Lorentz symmetry violation in the presence of a Coulomb-type scalar potential. The spectrum 
of energy Eq. (14) is symmetrical about $\varepsilon_{n}=0$ for constant or zero values of $l$ and are equally spaced on either side. We have established the background of the violation of the Lorentz symmetry defined by a tensor possessing the non-null components $\left\{\left(\kappa_{D E}\right)_{11}=\right.$ const, $\left(\kappa_{D B}\right)_{13}=$ const $\}$.

\section{Conclusions}

We have investigated the effects of Lorentz symmetry violation (LSV) on a relativistic scalar particle by solving the Klein-Gordon equation in the presence of an external potential proportional to the inverse of the radial distance $\left(\propto \frac{1}{r}\right)$. Though our work is inspired by the Standard Model Extension (SME) that is an effective field theory, we have relaxed the renormalization property in our analysis. We have shown that the solution of the bound state to the Klein-Gordon equation can be obtained under the effects of the Lorentz symmetry violation defined by a radial electric field produced by linear distribution of electric charge, a uniform magnetic field along the $z$-direction, and the dimensionless tensor $\left(K_{F}\right)_{\alpha \beta \mu \nu}$ that governs the Lorentz symmetry breaking possessing the non-null components $\left(\kappa_{D B}\right)_{13}=$ const, $\left(\kappa_{D E}\right)_{11}=$ const. After solving the radial wave equation, we have obtained the energy spectrum Eq. (14) of the bound state, and the wave function Eq. (15) of a relativistic scalar particle. We can see that the presence of the non-null components of the tensor $\left(K_{F}\right)_{\mu \nu \alpha \beta}$ that governs the Lorentz symmetry violation and the scalar potential modified the spectrum of energy Eq. (14) and the wave function (15).

\section{References}

[1] M. M. Ferreira Jr. and F. M. O. Moucherek, Nucl. Phys. A 790, 635 (2007). 
[2] L. R. Ribeiro, E. Passos and C. Furtado, J. Phys. G : Nucl. Phys. 39, 105004 (2012).

[3] H. Belich, E. O. Silva, M. M. Ferreira Jr., M. T. D. Orlando, Phys. Rev. D 83, 125025 (2011).

[4] K. Bakke and H. Belich, J. Phys. G : Nucl. Part. Phys. 39, 085001 (2012).

[5] K. Bakke, E. O. Silva and H. Belich, J. Phys. G : Nucl. Particle Phys. 39, 055004 (2012).

[6] K. Bakke and H. Belich, Ann. Phys. (N. Y.) 354, 1 (2015).

[7] R. Casana, M. M. Ferreira Jr., E. Passos, F. E. P. dos Santos and E. O. Silva, Phys. Rev. D 87, 047701 (2013).

[8] K. Bakke and H. Belich, Ann. Phys. (Berlin) 526, 187 (2014).

[9] K. Bakke and H. Belich, J. Phys. G : Nucl. Part. Phys. 42, 095001 (2015).

[10] K. Bakke and H. Belich, J. Phys. G : Nucl. Part. Phys. 40, 065002 (2013).

[11] A. G. de Lima, H. Belich and K. Bakke, Eur. Phys. J. Plus 128, 154 (2013).

[12] K. Bakke and H. Belich, Eur. Phys. J. Plus 127, 102 (2012).

[13] K. Bakke and H. Belich, Ann. Phys. (N. Y.) 373, 115 (2016).

[14] R. L. L. Vitoria, H. Belich and K. Bakke, Adv. High Energy Phys. 2017, 6893084 (2017).

[15] R. L. L. Vitoria, K. Bakke and H. Belich, Ann. Phys. (N. Y.) 399, 117 (2018). 
[16] K. Bakke and H. Belich, Ann. Phys. (N. Y.) 360, 596 (2015).

[17] R. L. L. Vitoria and H. Belich, Eur. Phys. J. C 78, 999 (2018).

[18] K. Bakke and H. Belich, Ann. Phys. (N. Y.) 333, 272 (2013).

[19] R. L. L. Vitoria, H. Belich and K. Bakke, Eur. Phys. J. Plus 132, 25 (2017).

[20] R. L. L. Vitoria and H. Belich, Eur. Phys. J. Plus 135, 247 (2020).

[21] K. Bakke and H. Belich, Int. J. Mod. Phys. A 30, 1550197 (2015).

[22] A. G. de Lima, H. Belich and K. Bakke, Ann. Phys. (Berlin) 526, 514 (2014).

[23] D. Colladay and V. A. Kostelecký, Phys. Rev. D 55, 6760 (1997).

[24] D. Colladay and V. A. Kostelecky, Phys. Rev. D 58, 116002 (1998).

[25] V. A. Kostelecky and R. Potting, Phys. Rev. D 51, 3923 (1995).

[26] V. A. Kostelecky and R. Potting, Phys. Lett. B 381, 89 (1996).

[27] S. Carroll, G. Field and R. Jackiw, Phys. Rev. D 41, 1231 (1990).

[28] A. P. B. Scarpelli, H. Belich, J. L. Boldo, L. P. Colatto, J. A. HelayelNeto and A. L. M. A. Nogueira, Nucl. Phys. (Proc. Suppl.) 127, 105 (2004).

[29] V. A. Kostelecky and M. Mewes, Phys. Rev. Lett. 87, 251304 (2001).

[30] V. A. Kostelecky and M. Mewes, Phys. Rev. D 66, 056005 (2002).

[31] V. A. Kostelecky and M. Mewes, Phys. Rev. Lett. 97, 140401 (2006).

[32] H. Belich, F. J. L. Leal, H. L. C. Louzada and M. T. D. Orlando, Phys. Rev. D 86, 125037 (2012). 
[33] Quentin G. Bailey and V. Alan Kostelecky, Phys. Rev. D 70, 076006 (2004).

[34] V. A. Kostelecký and N. Russell, Rev. Mod. Phys. 83, 11 (2011).

[35] H. G. Dosch, J. H. Jansen and V. F. Müller, Phys. Norv. 5, 2 (1971).

[36] E. R. F. Medeiros and E. R. Bezerra de Mello, Eur. Phys. J. C 72, 2051 (2012).

[37] M. Abramowitz and I. A. Stegum, Hand book of Mathematical Functions, Dover Publications Inc., New York (1965).

[38] G. B. Arfken and H. J. Weber, Mathematical Methods For Physicists, Elsevier Academic Press, London (2005). 